\title{
Role of tumor-derived exosomes in bone metastasis (Review)
}

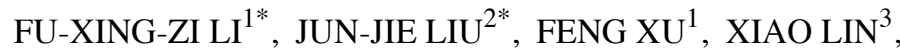 \\ JIA-YU ZHONG $^{3}$, FENG WU ${ }^{4}$ and LING-QING YUAN ${ }^{1}$ \\ ${ }^{1}$ Department of Endocrinology and Metabolism, Hunan Provincial Key Laboratory of Metabolic Bone Diseases, \\ National Clinical Research Center for Metabolic Disease, The Second Xiang-Ya Hospital; \\ ${ }^{2}$ Department of Pathology, The Xiangya Stomatological Hospital; \\ Departments of ${ }^{3}$ Geriatrics and ${ }^{4}$ Pathology, The Second Xiang-Ya Hospital, \\ Central South University, Changsha, Hunan 410011, P.R. China
}

Received November 29, 2018; Accepted May 5, 2019

DOI: $10.3892 / 01.2019 .10776$

\begin{abstract}
Tight coupling between bone resorption and formation is essential for bone remodeling. Disruption of this equilibrium can lead to skeletal disorders. Osseous metastatic disease is a severe consequence of tumor cell dissemination from numerous primary cancer sites, including the prostate, lungs and breasts. Metastatic disease is one of the most common causes of mortality in patients with cancer. Rapid advances in the therapeutic options for bone disease, including the use of bisphosphonates, have achieved effective clinical effects. However, the overall survival time of patients with bone metastatic has not significantly improved. Exosomes, which originate from tumor tissue and preferentially the bone, provide a reasonable way to understand the mechanism of neoplastic bone metastasis. Recently, several studies have indicated that tumor-derived exosomes are involved in cancer progression. However, the potential role that exosomes serve in the pathological communication between tumor and bone cells within the skeletal microenvironment remains an emerging field. The present review reports some recent findings on the detrimental roles of exosomes in bone metastasis. In addition, since exosomes are involved in metabolic organ cross-talk, this review highlights the involvement of cancer-derived exosomes in the regulation of skeletal metastatic diseases. Lastly, the potential promising clinical applications and emerging therapeutic opportunities targeting exosomes are discussed as novel strategies for cancer therapy.
\end{abstract}

Correspondence to: Professor Ling-Qing Yuan, Department of Endocrinology and Metabolism, Hunan Provincial Key Laboratory of Metabolic Bone Diseases, National Clinical Research Center for Metabolic Disease, The Second Xiang-Ya Hospital, Central South University, 139 Middle Renmin Road, Changsha, Hunan 410011, P.R. China

E-mail: allenylq@csu.edu.cn

${ }^{*}$ Contributed equally

Key words: exosomes, osteoblast, osteoclast, bone metastasis, pre-metastatic niche

\section{Contents}

1. Introduction

2. Exosomes and microRNA (miRNA/miR)

3. Cancer to the bone: A fatal attraction

4. Exosomes and the pre-metastatic niche

5. Tumor-derived exosomes and bone disease

6. Conclusions

\section{Introduction}

Malignant tumors have become the second-leading cause of mortality worldwide, and it has been predicted that the number of newly diagnosed malignancies will increase to 23.6 million by 2030 (1). The transfer of tumor cells to distant metastases is the most common cause of cancer-associated mortality (2). The bone is a major target for cancer metastasis, second only to the lungs and liver; it has unique anatomical and physiological pathological conditions, which facilitate cancer metastasis, especially from solid tumors (3), including those of the prostate, breasts, kidneys and lungs (4). Firstly, tumor cells themselves have the ability to migrate from the primary lesion to distant skeletal tissue; they can also accelerate tumor growth, infiltrate the surrounding tissues and cause distance metastases, which are all associated with tumor heterogeneity. Secondly, the anatomical characteristics of the skeletal system are unique. For example, the red bone marrow allows tumor cells to enter the bloodstream and remain within the bone marrow tissue (5). Thirdly, affected bones and metastatic tumor cells cause various types of biological response, including adhesion molecules produced by tumor cells that bind to trabecular bone and stromal cells. Finally, tumor cells further produce angiogenic factors and bone resorption factors (6). In addition, hematological tumors might directly or indirectly affect the metabolism of bone. However, these cancer metastases remain incurable, and the 5-year survival rate of patients with these types of metastasis is significantly reduced (7). This results in a series of complications, including severe bone pain, pathological fractures, hypercalcemia and spinal cord compression, which severely affects the quality 
of life and the life expectancy of patients. Highly specific interactions between disseminating cancer cells and the bone microenvironment determine the metastatic process $(4,8)$. The equilibrium between the activity of bone resorbing osteoclasts and bone-forming osteoblasts is interrupted by bone metastasis. Due to the abundant blood supply and special growth microenvironment in bone tissue (9), such permissive environments (pre-metastatic niche formation) are in favor of metastatic development (10-12). Cells can communicate with each other by secreting extracellular vesicles (EVs). These EVs display a diverse range of sizes. The present review specifically highlights the role of tumor-derived exosomes (40-100 nm diameter) in bone metastasis.

\section{Exosomes and microRNA (miRNA/miR)}

Exosomes are small disc-shaped vesicles, with a diameter of 40-100 nm, that contain mRNAs, miRNAs (13), lipids $(14,15)$ and proteins (14). Exosomes are secreted by a wide variety of normal and malignant cells (16), formed by the endosomal network and released from the cell via the fusion of multi-vesicular bodies with the plasma membrane $(17,18)$. Exosomes are characterized by specific markers, including CD9, CD63, CD81, Alix and TSG101 $(13,14)$. The role of exosomes in intercellular communication, via the transfer of proteins (19), bioactive lipids and miRNAs (20), has been confirmed in numerous studies (21). Exosomes are found in almost all body fluids, including the serum (22), saliva (23), breast milk (24), cerebrospinal fluid (25), urine (14) and semen (26). Furthermore, exosomes are largely found in the tumor microenvironment. Tumor-derived exosomes have received considerable attention for their role in cancer progression and metastasis, and previous studies reported that they serve a pivotal role in cancer growth, development and metastasis (27-32). A recent study revealed that the amount and contents of the exosomes secreted by tumor cells are much larger compared with that of normal cells (33). The variable exosome contents therefore influence their behavior and strongly modify the entire microenvironment (34). Exosomes significantly contribute to the communication between cells and the subsequent reprogramming of the tumor microenvironment (35). Exosomes primarily promote tumor metastasis as follows: i) Exosomes secreted by tumor cells directly open the way for tumor invasion and metastasis (36); ii) tumor cells with high metastatic potential can promote the invasion and metastasis of tumor cells themselves, or other relatively low metastatic potential tumor cells through exosomes (30); iii) the cross-talk between mesenchymal cells and tumor cells via exosomes ultimately promotes tumor metastasis (37); and iv) tumor-derived exosomes regulate mesenchymal cells in the distant metastasis microenvironment to promote distant colonization and proliferation (10). Numerous specific tumor cells, including those of the prostate, lungs and breasts, are more prone to bone metastasis and have substantial crosstalk with bone cells in the bone microenvironment (38). Whether tumor-derived exosomes are involved in interactions between tumor and bone cells, and the underlying mechanisms of such communication, remain unclear.

Long non-coding RNAs and miRNAs constitute a class of small (19 to 25-nucleotide) RNAs that serve crucial gene regulatory roles in humans (39). Extensive research has revealed that miRNAs have regulatory roles in a wide range of pathological and physiological processes $(13,40)$. For example, Van Balkom et al (41) reported that human microvascular endothelial cell (EC)-derived exosomes, containing miR-214, promoted EC migration and angiogenesis. Furthermore, knockout of miR-214 in ECs resulted in loss of the ability of EC-derived exosomes to promote migration and angiogenesis. Cui et al (42) reported that mouse embryonic osteogenic precursor cells secreted a variety of Wnt/ $\beta$-catenin signaling pathways that activate bone marrow mesenchymal stem cells (BMSCs) and osteogenesis-associated miRNAs during osteogenic differentiation. Exosomes upregulated the expression of the osteogenesis-associated genes and promoted the formation of mineralized nodules. These findings suggest that these miRNAs can be transferred to effector cells through exosomes to exert their gene regulatory functions, by enriching certain miRNAs in the source cells. Kumar and Reddy (20) reported that exosomes secreted by cells in disease states contain mainly disease-specific or deregulated miRNAs, and that they can be used as diagnostic molecules. A previous study also demonstrated that exosomes from the plasma of patients with various types of cancer present with distinct miRNA signatures (43). However, these characteristics do not correspond to those from the parent tumor cell (44), which suggests that exosomes selectively release miRNA from tumor cells. Growing evidence indicates that some exosomes isolated from cancer patients have distinct miRNA profiles, including those of lung cancer (45) and breast carcinoma (46), which suggests that these miRNAs might be considered as specific diagnostic markers for patients with cancer.

\section{Cancer to the bone: A fatal attraction}

'Seed and soil' doctrine. Over 100 years ago, Stephen Paget proposed the 'seed and soil' doctrine, suggesting that tumor metastasis was not random and emphasizing the interaction between tumor cells and target tissues, proposing that cancer cells were like 'seeds' and that the bone microenvironment was like the 'soil' (47). The environment provides the necessary nutritional support for cancer cells, which have an affinity with the bone microenvironment (48). Tumor invasion into the bone is associated with the recruitment of osteoclasts and osteoblasts, resulting in the release of growth factors that accumulate in the bone matrix. This phenomenon eventually induces positive feedback for further tumor growth, and can be considered as a 'vicious circle' of bone metastasis $(49,50)$. Simultaneously, bone marrow also serves as a repository for dormant tumor cells that are resistant to chemotherapy, and these cells can then be transferred to the bone or other organs $(51,52)$.

Osteolytic bone metastases and osteoblastic metastases. Normal bone homeostasis depends on osteoblastic bone formation and osteoclastic bone resorption (53). Bone metastasis is a complex cascade of processes (54). Firstly, tumor cells have a tendency to travel into the bone through specific migration and invasion processes. Secondly, these tumor cells gain bone-like properties and reach the bone marrow. Finally, tumor cells interact with osteoclasts and osteoblasts. This 
interaction determines whether subsequent bone metastases become osteolytic or osteogenic. Clinically, 65 to $70 \%$ patients with bone metastases exhibit osteolytic metastasis. Previous studies reported that tumor-derived microvesicles, known as exosomes, facilitate the initial communication between the primary tumor and the metastatic site $(43,55)$. Cancer cell metastasis to bone tissue results in osteolytic destruction. This phenomenon is not only caused by the direct effect of cancer cells on bone cells, but also through the secretion of cytokines that interact with the bone microenvironment, which results in osteoclast activation and subsequent bone destruction (41). This vicious cycle between cancer cells and the bone microenvironment results in tumor cell proliferation and continuous bone mass destruction. Receptor activator for nuclear factor- $\kappa \mathrm{B}$ ligand (RANKL), which is a member of the tumor necrosis factor family, is expressed and released by osteoblasts and BMSCs. Parathyroid hormone-related protein (PTH-rP) secreted by cancer cells directly stimulates osteoblasts to secrete RANKL (42). RANKL and macrophage colony-stimulating factors signaling molecules, which are necessary for the differentiation of osteoclast precursors into osteoclasts. Once bone metastasis occurs, cancer cells can secrete cytokines that enhance RANKL expression, which inhibits osteoblast function and expression of other tumor-associated cells, including fibroblasts, immune cells and osteoprotegerin (OPG). As a result, RANKL enhances osteoclast activity, which leads to bone destruction, and causes increases in the levels of insulin-like growth factors (IGFs), transforming growth factor- $\beta$ (TGF- $\beta$ ), fibroblast growth factors (FGFs) and other cytokines released in the bone matrix that further stimulate tumor growth (56). In addition, osteolytic lesions increased extracellular calcium $\left(\mathrm{Ca}^{2+}\right)$ concentration, which stimulates PTH-rP secretion, resulting in increased osteoclast activity and the formation of the aforementioned vicious cycle (57).

Osteoblastic metastases are characterized by increased pathological osteogenesis. These new bones do not have the function of normal bone and destroy the normal structure of healthy bone. Vascular endothelial growth factor (VEGF), platelet-derived growth factor (PDGF) and endothelin-1 (ET-1) are secreted by tumor cells themselves, which can stimulate osteoblast proliferation (58-60). ET-1 serves an important role in the formation of bone metastases in prostate cancer. This protein has a dual role in stimulating proliferation and activation of prostate cancer cells and osteoblasts (61). In addition, ET-1 stimulates not only the growth of prostate cancer cells but also the response to growth factors, including IGF and PDGF (Fig. 1) (62).

Current status of treatment for bone metastatic carcinoma. The morbidity and mortality rates of bone metastatic carcinoma are persistently high, which severely affects the survival time and quality of life of patients with bone metastases (63). Targeted therapy for bone metastasis aims to reduce or delay the occurrence of bone-associated events, including pathological fracture and bone pain, therefore improving the quality of life of patients and extending their survival time. Advances in the therapeutic options for the treatment of bone disease include the use of bisphosphonates (64), denosumab (65) or RANKL-antibodies that target osteoclastogenesis, which have significantly reduced the complications of bone metastasis and offered good clinical effects (66-68). However, the overall survival time of patients with bone metastases has not significantly improved with these treatments (69). Inhibiting the occurrence of bone metastases, in particular in patients with extremely severe bone pain, therefore remains a major challenge for clinicians.

\section{Exosomes and the pre-metastatic niche}

The 'pre-metastatic niche' is a supportive microenvironment providing nutritional supplies for tumor cells before they metastasize from the primary organ to the distal organ (70). It has been demonstrated that BMSCs are crucial for the generation of an appropriate microenvironment for the primary tumor, and for the development of metastasis (71), through the process of pre-metastatic niche formation $(72,73)$. Previous studies have demonstrated that the serum of patients with cancer contains high exosome levels, which are positively correlated with the malignant behavior of the cancer (74-77). Tumor cells affect surrounding cells through direct contact, paracrine secretion, self-secretion, and by direct cross-talk via exosomes. This newly discovered cell interaction via exosomes serves an important role in tumor metastasis and invasion (78). Although some secreted factors recruit BMSCs to both the primary tumor and the pre-metastatic niche (79-81), studies about the role of exosomes in bone metabolism and the bone microenvironment have been limited until recently. Thanks to the emergence of fluorescence exosome-labeling technology and the determination of specific markers of exosomes, the research on the association between exosomes and endothelial cells, angiogenesis and metastatic promotion has made significant advances (70). Methods for identification and characterization of exosomes include the observation of morphology by transmission electron microscopy, the measurement of diameter by dynamic light scattering, and the analysis of characteristic surface marker proteins by flow cytometry or western blot analysis. Furthermore, fluorescent dye kits for general cell or exosome membrane labeling, such as green fluorescent dyes PKH67 (14) or red fluorescent dyes PKH26 (82) (both Sigma-Aldrich; Merck KGaA) can be used to label exosomes in vitro. 1,1-dioctadecyl-3,3,3'3'-tetramethylindotricarbocyanine-iodide (83) fluorescent dye can be used to label exosomes in vivo. A previous study reported that the systemic delivery of fluorescent exosomes from metastatic B16F10 melanoma cells localizes to the lungs and other tissues, including the bone (84). Tumor-derived exosomes are important mediators of tumorigenesis that are able to educate stem cells for neoplastic transformation and tumor metastasis (50). Exosomes from highly metastatic melanomas have been reported to increase the in vivo metastatic behavior of primary tumors via permanently 'educating' bone marrow progenitors, via MET receptor tyrosine kinase upregulation in bone marrow cells. Notably, this reprogramming effect of exosomes on BMSCs is enduring, which may explain how tumors that have been dormant for decades suddenly develop metastatic disease (84). This research may help to clarify the 'seed and soil' hypothesis, and to determine the mechanism of organ-specific transfer theory (47). In the 'seed and oil' theory, exosomes may be the real seeds of cancer. Hoshino et al (85) revealed that tumor exosome integrins can establish a pre-metastasis 


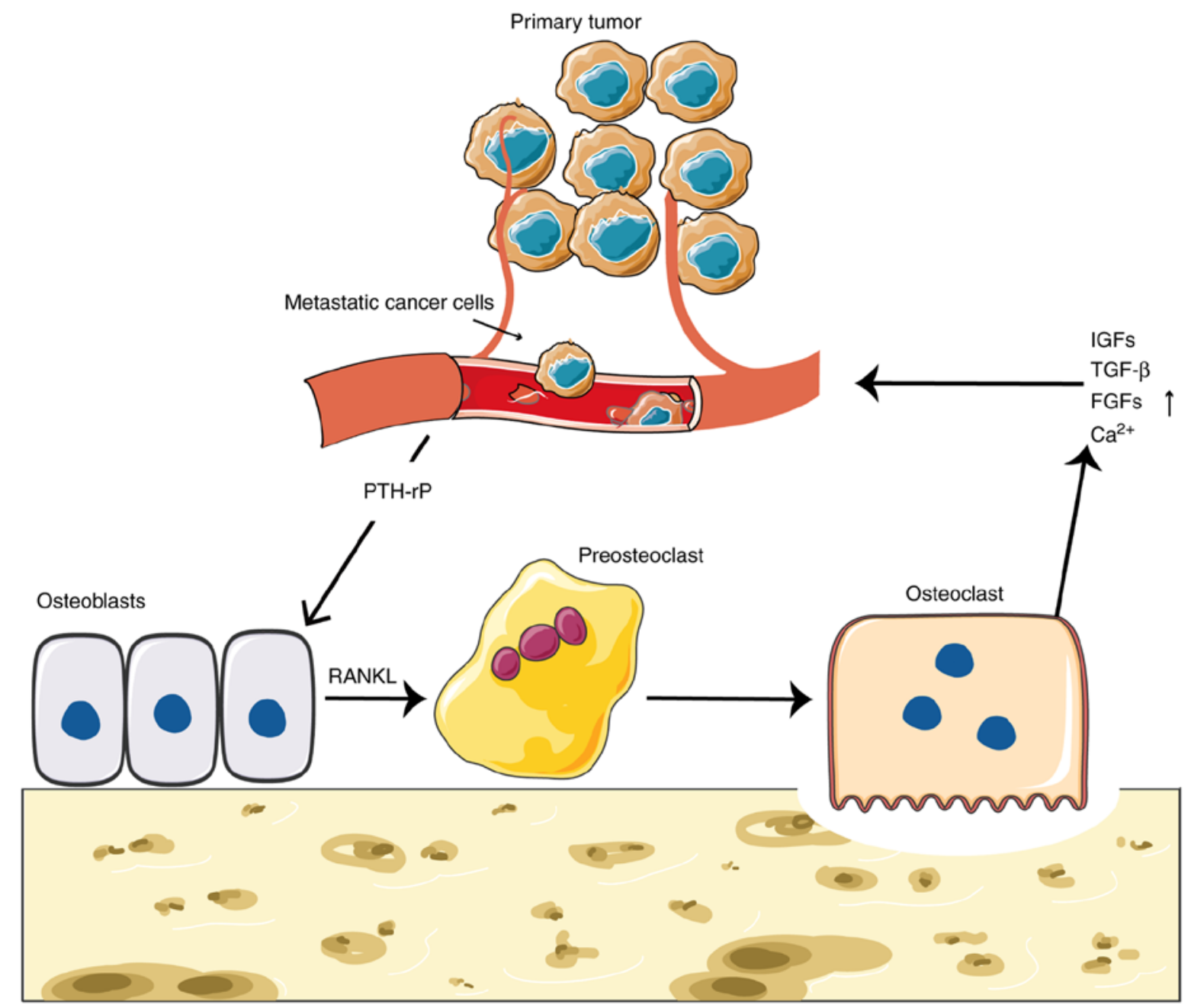

Figure 1. Complex vicious cycle of bone metastasis involving mutual interactions between tumor cells, bone cells (osteoclasts and osteoblasts) and the bone matrix. The majority of tumor cells have a tendency to travel into the bone through blood vessels. These cells secrete PTH-rP, which stimulates osteoblasts to secrete RANKL. The expression of RANKL is necessary for the differentiation of osteoclast precursors into osteoclasts. As a result, RANKL activates osteoclasts, which leads to bone destruction, and causes increases in the levels of IGFs, TGF- $\beta$, FGFs and other cytokines that are released in the bone matrix. In addition, osteolytic lesions increase the concentration of $\mathrm{Ca}^{2+}$. Subsequently, all these factors stimulate osteoclast activity and tumor growth. The complex interaction between the bone microenvironment and tumor cells leads to the so-called 'vicious cycle'. FGFs, fibroblast growth factors; IGFs, insulin-like growth factors; PTH-rP, parathyroid hormone-related protein; RANKL, receptor activator for nuclear factor- $\mathrm{B}$ ligand; TGF- $\beta$, transforming growth factor- $\beta$.

microenvironment by organ-specific colonization, and that they can therefore determine organ-specific cancer metastasis. In this study, $\sim 10$ different tumors were analyzed and the levels of $\sim 1,000$ proteins in the exosomes were determined, looking for key proteins that could be a special 'zip code'. Exosomes can be considered as 'signal vessels', and integrins, which are closely associated with cancer in the lung and liver metastasis, are present at the surface of exosomes. This special 'labeling' of exosomes may allow them to enter specific organs and continuously accumulate to further promote metastasis. This study also demonstrated that, when treated with lung metastatic tumor-derived exosomes (such as those from breast cancer cells), metastatic tumor cells that are prone to metastasize to the bone are no longer transferred to this region, but are instead redirected to the lungs. This finding suggests that the metastatic characteristics of tumor cells are not autonomous and are influenced by external factors. In addition, exosomes that target different organs have distinctive cell adhesion receptor proteins and cell-surface integrins. Exosomes have a tendency to enter organs with large numbers of ligands corresponding to their surface integrins. In conclusion, exosomes appear to serve a crucial role in the establishment of the pre-metastatic niche. These findings provide some directions for the identification of novel anticancer targets in the later stage, and for the development of novel anticancer therapies.

\section{Tumor-derived exosomes and bone disease}

Recently, there has been growing interest in the cell-cell communication roles of exosomes in cancer. Tumor-derived exosomes serve a crucial role in cancer survival, apoptosis, invasion, angiogenesis and resistance to chemotherapy; they are also involved in the establishment of the metastatic niche (86). Numerous specific tumor cells, including those of the prostate, lungs and breasts, are prone to bone metastasis and have substantial cross-talk with bone cells in the bone microenvironment (Fig. 2).

Multiple myeloma (MM). Increased osteoclastic activity is a major element of bone disease in MM (87-90). The increased number and activity of osteoclasts further promote the 
Multiple myeloma
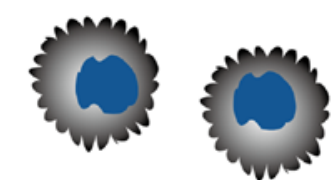

$\downarrow$

Exosomes

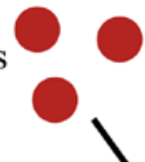

Exosomes
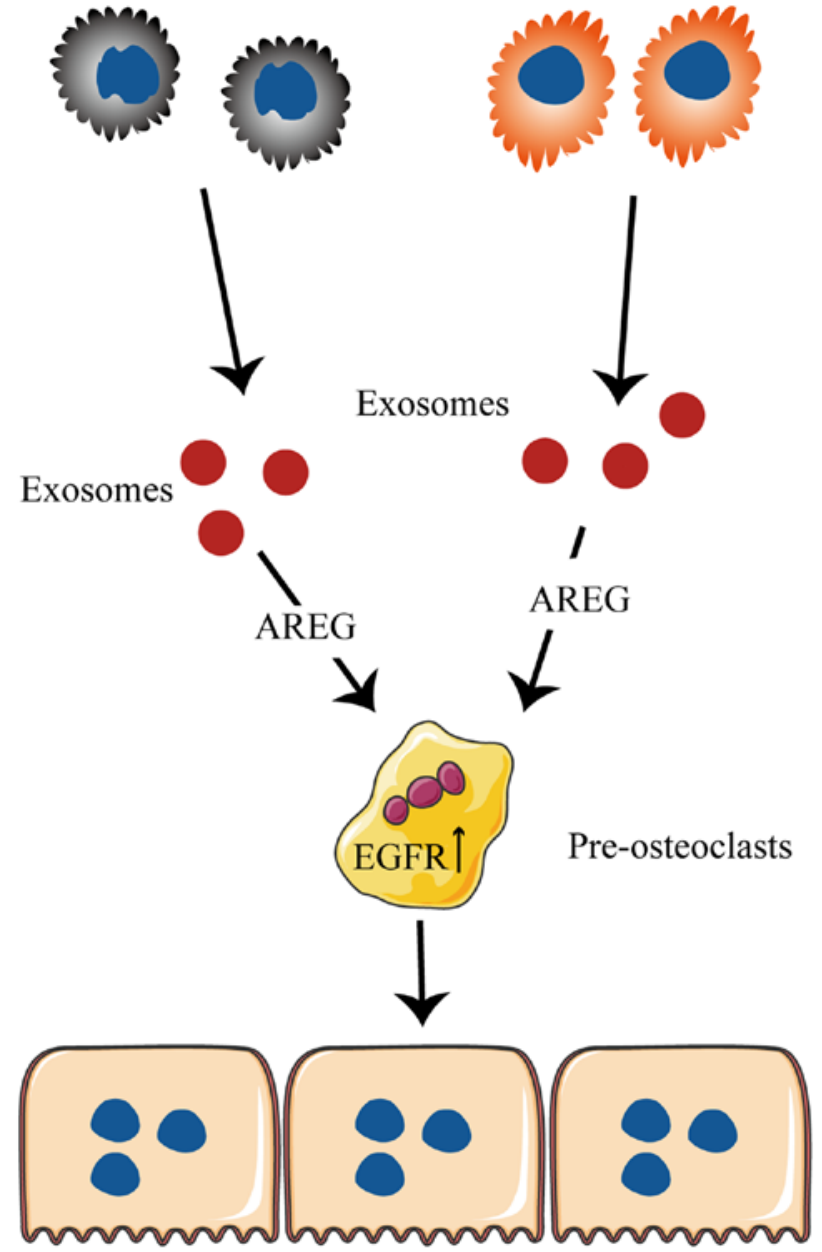

Osteoclasts
Prostate cancer

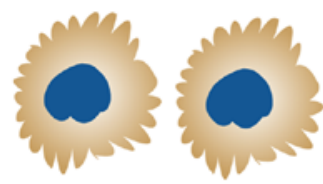

$\downarrow$

Exosomes

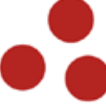

ETS1, hsa-miR-940, miR-141-3p

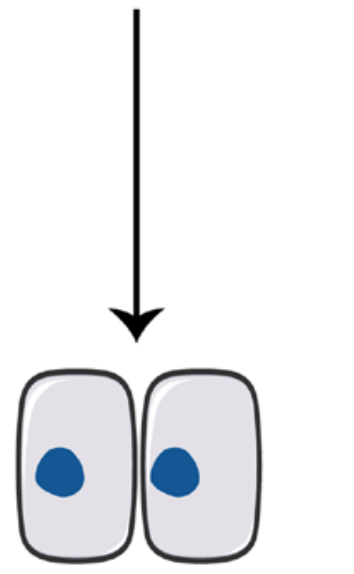

Osteoblasts

Figure 2. Exosomes secreted by MM, NSCLC and PCa tumors carry different and abundant contents, which serve a key role in osteoclasts or osteoblasts, resulting in different metastatic lesions. AREG in MM-derived exosomes leads to the activation of EGFR in pre-osteoclasts, participating in MM-induced osteoclastogenesis. NSCLC-derived exosomes containing AREG induce EGFR pathway activation in pre-osteoclasts. PCa-derived exosomes contain Ets1, hsa-miR-940 and miR-141-3p, which ultimately lead to extensive osteoblastic lesions. AREG, amphiregulin; EGFR, epidermal growth factor receptor; miR, microRNA; MM, multiple myeloma; NSCLC, non-small cell lung cancer; PCa, prostate cancer.

progression of MM via direct and indirect mechanisms, thus maintaining a vicious cycle between bone destruction and tumor cell survival. Furthermore, Raimondi et al (91) indicated that MM-derived exosomes have a positive role in modulating migration and pro-differentiation of the pre-osteoclast. In addition, MM-derived exosomes induce osteoclast formation and promote bone resorption in the mature osteoclast-like cell. Moreover, Garimella et al (92) demonstrated that the highly aggressive and metastatic osteosarcoma 143B cells produce exosomes primarily through mechanisms that actively mobilize intracellular calcium or cyclic adenosine monophosphate (cAMP) levels, in the presence of ionomycin and forskolin, thereby increasing intracellular calcium or cAMP levels. Raimondo et al (93) reported that amphiregulin (AREG) is specifically enriched in exosomes from MM samples and that exosome-derived AREG lead to the activation of epidermal growth factor receptor ligands (EGFR) in pre-osteoclasts, participating in MM-induced osteoclastogenesis. These results suggest that exosomes are important mediators of the cross-talk between MM cells and the bone marrow microenvironment. These data further support the role of tumor cell-derived microvesicles in cancer progression and disease aggressiveness.

Prostate cancer (PCa). Bone metastases are common in patients suffering from $\mathrm{PCa}$ (94). The recent emergence of exosomes provides a novel explanation for bone metastasis in $\mathrm{PCa}$. Bone metastases in PCa can be categorized as osteogenic or osteolytic metastases. Exosomes from PCa may therefore affect osteoblasts and osteoclasts. Karlsson et al (95) reported that exosomes isolated from PCa cells markedly decreased the fusion and differentiation of monocytic osteoclast precursors into mature and multinucleated osteoclasts. The presence of tumor cell-derived exosomes also clearly decreased the expression of the established osteoclast fusion and differentiation markers, including dendritic cells-specific transmembrane proteins, transmembrane AMPAR regulatory proteins, cathepsin $\mathrm{K}$ and matrix metalloproteinase-9 (95). A study by Inder et al (96) indicated that exosomes isolated from the conditioned medium 
of the PC3 cell line were internalized into osteoclast precursors and osteoblasts, which stimulated osteoclastogenesis and osteoblast proliferation 37- and 1.5-fold, respectively; however, EVs derived from cavin-1-expressing PC 3 cells failed to induce multinucleate osteoblast or human osteoblast proliferation. Cavin-1 was not detected in EVs, indicating an indirect mechanism of action. Furthermore, it was demonstrated that cavin-1 was not present in the vesicles, which revealed that cavin-1 modulated cargo vesicle recruitment rather than release (96). A vicious cycle has also been established between PCa cells and osteoblasts. In osteoblastic metastasis, the osteoblastic microenvironment acts as a pre-metastatic niche by attracting bone-metastasizing tumors (97). In the past few years, research has indicated that PCa cells provide osteoblasts with osteogenic cytokines (BMPs, PDGF, ET-1 and VEGF) and supply osteolytic factors (TGF- $\beta$ and IGFs) to osteoclasts, which indicates that these PCa cells communicate with each other and thereby regulate tumor growth in a careful and complex manner (98). However, the effects of PCa-derived exosomes on osteoblast function remain unclear. Itoh et al (99) isolated exosomes from hormone refractory PCa cells, and reported that PCa-derived exosomes prepared from either PC3 or DU145 cell cultures significantly facilitated osteoblast differentiation; however, $\mathrm{PCa}$-derived exosomes from LNCaP cells did not have this effect. The subsequent study further indicated that higher expression levels of Ets1, which is an osteoblast differentiation-associated transcriptional factor, is a potential candidate than can induce osteoblast differentiation. Hormone refractory PCa cell-derived exosomes containing Ets1 were transferred into osteoblasts, and Ets1 was released into the cytoplasm and induced differentiation (99). Ye et al (100) demonstrated that exosomal miR-141-3p increased OPG expression, which resulted in a significant increase in OPG/RANKL levels. Furthermore, miR-141-3p suppressed the protein levels of the DLC1 target gene in vitro through activation of the p38/MAPK signaling pathway. In addition, in animal experiments, miR-141-3p specifically targeted the bones and promoted osteoblast activity. Recently, Hashimoto et al (101) revealed the overexpression of hsa-miR-940 in tumor exosomes and its ability to induce the osteogenic differentiation of host mesenchymal cells, ultimately leading to extensive osteoblastic lesions in the resulting tumor. Morhayim et al (102) demonstrated that human osteoblasts-derived exosomes could promote PCa cell proliferation in vitro. Cancer cell-derived exosomes therefore mediate cell-cell communication in osteoblastic metastasis, and osteoblast-derived exosomes may regulate cancer cell proliferation in the metastatic microenvironment. Pro-metastatic exosomes represent a novel way for osteoclasts or osteoblasts to communicate with cancer cells, and may offer a novel tool for therapeutic intervention in patients with cancer.

Non-small cell lung cancer (NSCLC). NSCLC cells release factors that alter bone remodeling and increase osteoclast activity through a shift in the normal balance of RANKL and OPG (103). In a study by Taverna et al (104), it was demonstrated that NSCLC-derived exosomes that contain AREG could induce EGFR pathway activation in pre-osteoclasts through activation of EGFR phosphorylation, which subsequently causes the increased expression of RANKL. This phenomenon induces the increase of MMP9 and TRAP expression, which triggers a vicious cycle in osteolytic bone metastasis. A previous study by Valencia et al (105) demonstrated that miR-192 elicits pleiotropic functions that cooperatively attenuate osseous metastasis. This alters the cargo of cancer cell-derived exosomes via the overexpression of a single anti-angiogenic miRNA (miR-192), and represses the tumor-induced angiogenesis, which leads to a reduction in the number of bone metastatic lesions in mice. Targeting one or more miRNAs may therefore represent a potentially beneficial strategy to block the metastatic process. However, changing the miRNA-cargo content in exosomes could represent a novel mechanism that may have a large positive impact on bone metastases.

Acute myelocytic leukemia (AML). The ability of malignant blood cells to transform the blood microenvironment niche remains currently unknown. Kumar et al (106) recently revealed a novel mode of niche transformation. The study demonstrated that acute leukemia cells are able to change the hematopoietic microenvironment, and can transform a normal niche into a malignant niche through the transfer of exosomes [via dickkopf WNT signaling pathway inhibitor 1 (DKK1) gene], which provides a suitable environment for their proliferation. Furthermore, the grafting or injection of AML-derived exosomes can increase mesenchymal progenitor cells and block osteogenesis and bone formation in the body. In addition, they can accelerate AML cell proliferation. Conversely, AML-derived exosomes can be destroyed by the targeted inhibition of Rab27a, which can significantly delay the development of leukemia (106). Since DKK1 is a normal hematopoietic and osteogenic inhibitory factor, subsequent studies demonstrated that AML-derived exosomes stimulate DKK1 to cause osteocyte loss (106). Targeting exosomes may therefore represent a novel strategy in cancer therapy. Effective inhibition of the hematopoietic microenvironment may be an important new direction to control the growth of malignant blood cells.

Together, these studies demonstrated that tumor-derived exosomes serve a role in the cross-talk between osteoclasts and tumor cells, which highlights the importance of exosome cargo in cancer regulation. Furthermore, these findings significantly enhance our understanding of intercellular communication in bone metastasis by demonstrating that tumor cells release biologically active exosomes that are responsible inside the metastatic niche for the recruitment, migration and differentiation of osteoclast precursors through re-expression of miRNA inhibition. In addition, these studies highlight the role of cargo contained in tumor-derived exosomes in osteoclast differentiation, which may allow the development of novel therapeutic strategies to inhibit the fatal attraction between cancer and bone. The roles of the different tumor-derived exosomes in bone constituting development and progression are summarized in Table I. Breast cancer can also easily translocate into the bones (107); however, the role of breast cancer cell-derived exosomes in bone metastasis has not yet been reported.

\section{Conclusions}

Bone microenvironment facilitates tumor-induced bone destruction, and reduction of bone mass and strength. Tumor cells have developed numerous mechanisms to counteract the effects of chemotherapy drugs and prevent their elimination 


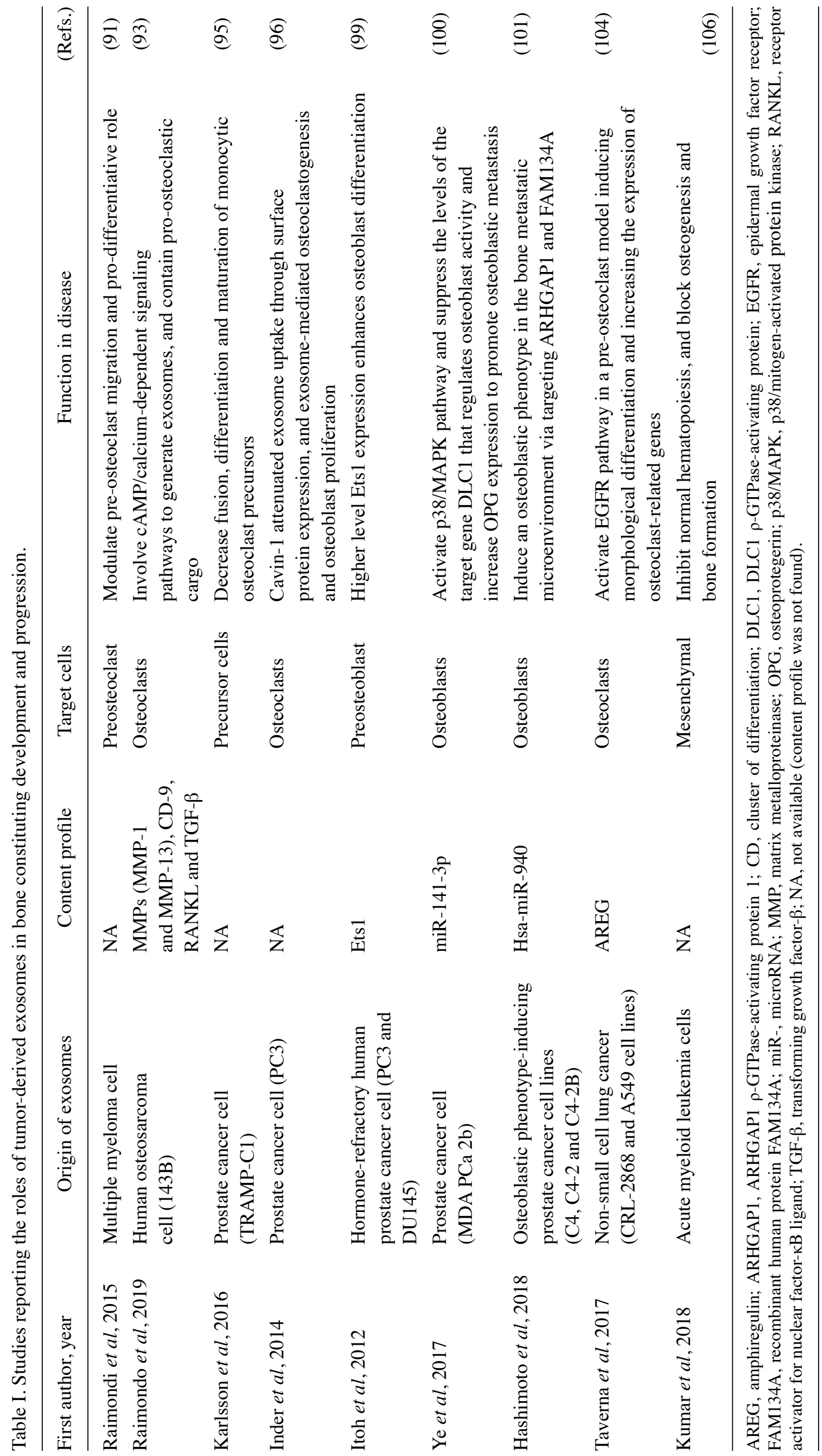


from the organism. One of these mechanisms involves highly specific exosomes. Exosomes serve a crucial role in the regulation of the local microenvironment surrounding the tumor and in cell-cell communication. However, the components of cell-derived exosomes require further investigation. The underlying mechanisms of tumor-derived exosomes in bone metastasis are not yet fully understood.

Although there is debate among experts, in general, tumor-derived exosomes have more deleterious effects than beneficial effects as tumor-secreted vesicles. The exosomes can stimulate tumor growth and development, and promote the process of bone metastasis. The role of exosomes in cancer progression and metastasis has drawn increasing attention, with studies essentially focused on their potential role as biomarkers and targets. Emerging evidence on exosome functions in bone metastasis may allow the discovery of novel ways to treat bone metastases. Overall, the results of therapies focusing on tumor-derived exosomes are discouraging. These exosomes usually carry numerous tumor activator molecules, which subsequently stimulate tumor growth and metastasis, induce host immunosuppression. Thus, the inhibition of exosome secretion may play a role in anticancer therapy. Tumor-derived exosomes therefore lead to cancer treatment failure, and the elimination of these exosomes seems to be applicable to the tumor and its metastatic treatment.

Tumor metastasis mechanisms are very complex and involve factors such as tumor cells, osteoblasts, osteoclasts and the bone microenvironment. Positive outcomes of anticancer treatment are therefore difficult to obtain. Further research on bone metastasis mechanisms will thus establish new experimental models, which could lead to more investigation on metastasis-associated factors, including exosomes and metastasis mechanisms. However, certain issues remain and need to be solved in the future, including the functions of components tumor-derived exosomes, including, RNA and DNA, in determining bone-specific metastasis, whether inhibiting the secretion of tumor-derived exosomes can prevent tumor metastasis, in particular bone metastasis, and other functional damage to the body, and how to translate future findings into clinical applications. Addressing these questions will help highlight the underlying mechanisms of bone metastasis. Future therapeutic strategies may involve the combination of several drugs that could block multiple targets or pathways at the same time, in order to improve quality of life, increase survival time and provide a greater therapeutic benefit for patients with bone metastases.

\section{Acknowledgements}

Not applicable.

\section{Funding}

This study was supported by the Fundamental Research Funds for the Central Universities of Central South University (grant no. 2018zzts918).

\section{Availability of data and materials}

Not applicable.

\section{Authors' contributions}

FXZL and JJL drafted the manuscript. FX, XL, JYZ, and FW were responsible for the collection of the relevant literature. LQY designed the outline and revised the manuscript. All authors have read and approved the final manuscript.

\section{Ethics approval and consent to participate}

Not applicable.

\section{Patient consent for publication}

Not applicable.

\section{Competing interests}

The authors declare that they have no competing interests.

\section{References}

1. Bray F, Jemal A, Grey N, Ferlay J and Forman D: Global cancer transitions according to the Human Development Index (2008-2030): A population-based study. Lancet Oncol 13: 790-801, 2012.

2. Gupta GP and Massagué J: Cancer metastasis: Building a framework. Cell 127: 679-695, 2006.

3. Mundy GR: Metastasis to bone: Causes, consequences and therapeutic opportunities. Nat Rev Cancer 2: 584-593, 2002.

4. Weilbaecher KN, Guise TA and McCauley LK: Cancer to bone: A fatal attraction. Nat Rev Cancer 11: 411-425, 2011.

5. Lalle M, De Rosa L, Marzetti L and Montuoro A: Detection of breast cancer cells in the bone marrow or peripheral blood: Methods and prognostic significance. Tumori 86: 183-190, 2000.

6. Van Der Pluijm G, Sijmons B, Vloedgraven H, Deckers M, Papapoulos S and Löwik C: Monitoring metastatic behavior of human tumor cells in mice with species-specific polymerase chain reaction: Elevated expression of angiogenesis and bone resorption stimulators by breast cancer in bone metastases. J Bone Miner Res 16: 1077-1091, 2001.

7. Jemal A, Siegel R, Xu J and Ward E: Cancer statistics, 2010. CA Cancer J Clin 60: 277-300, 2010.

8. Van Driel M and Van Leeuwen JP: Cancer and bone: A complex complex. Arch Biochem Biophys 561: 159-166, 2014.

9. Lynch ME and Fischbach C: Biomechanical forces in the skeleton and their relevance to bone metastasis: Biology and engineering considerations. Adv Drug Deliv Rev 79-80: 119-134, 2014.

10. Costa-Silva B, Aiello NM, Ocean AJ, Singh S, Zhang H, Thakur BK, Becker A, Hoshino A, Mark MT, Molina H, et al: Pancreatic cancer exosomes initiate pre-metastatic niche formation in the liver. Nat Cell Biol 17: 816-826, 2015.

11. Sceneay J, Parker BS, Smyth MJ and Möller A: Hypoxia-driven immunosuppression contributes to the pre-metastatic niche. Oncoimmunology 2: e22355, 2013.

12. Sceneay J, Smyth MJ and Möller A: The pre-metastatic niche: Finding common ground. Cancer Metastasis Rev 32: 449-464, 2013.

13. Hu Y, Rao SS, Wang ZX, Cao J, Tan YJ, Luo J, Li HM, Zhang WS, Chen CY and Xie H: Exosomes from human umbilical cord blood accelerate cutaneous wound healing through miR-21-3p-mediated promotion of angiogenesis and fibroblast function. Theranostics 8: 169-184, 2018.

14. Chen CY, Rao SS, Ren L, Hu XK, Tan YJ, Hu Y, Luo J, Liu YW, Yin H, Huang J, et al: Exosomal DMBT1 from human urine-derived stem cells facilitates diabetic wound repair by promoting angiogenesis. Theranostics 8: 1607-1623, 2018.

15. Record M, Silvente-Poirot S, Poirot M and Wakelam MJO: Extracellular vesicles: Lipids as key components of their biogenesis and functions. J Lipid Res 59: 1316-1324, 2018.

16. Skog J, Würdinger T, van Rijn S, Meijer DH, Gainche L, Sena-Esteves M, Curry WT Jr, Carter BS, Krichevsky AM and Breakefield XO: Glioblastoma microvesicles transport RNA and proteins that promote tumour growth and provide diagnostic biomarkers. Nat Cell Biol 10: 1470-1476, 2008. 
17. Sun Y and Liu J: Potential of cancer cell derived exosomes in clinical application: A review of recent research advances. Clin Ther 36: 863-872, 2014.

18. Yáñezmó M, Siljander PR, Andreu Z, Zavec AB, Borràs FE Buzas EI, Buzas K, Casal E, Cappello F, Carvalho J, et al: Biological properties of extracellular vesicles and their physiological functions. J Extracell Vesicles 4: 27066, 2015.

19. Zeelenberg IS, Ostrowski M, Krumeich S, Bobrie A, Jancic C Boissonnas A, Delcayre A, Le Pecq JB, Combadière B Amigorena $\mathrm{S}$ and Théry $\mathrm{C}$ : Targeting tumor antigens to secreted membrane vesicles in vivo induces efficient antitumor immune responses. Cancer Res 68: 1228-1235, 2008.

20. Kumar S and Reddy PH: Are circulating microRNAs peripheral biomarkers for Alzheimer's disease? Biochim Biophys Acta 1862: 1617-1627, 2016.

21. Valadi H, Ekström K, Bossios A, Sjöstrand M, Lee JJ and Lötvall JO: Exosome-mediated transfer of mRNAs and microRNAs is a novel mechanism of genetic exchange between cells. Nat Cell Biol 9: 654-659, 2007.

22. Li JJ, Wang B, Kodali MC, Chao C, Kim E, Patters BJ, Lan L, Kumar S, Wang X, Yue J and Liao FF: In vivo evidence for the contribution of peripheral circulating inflammatory exosomes to neuroinflammation. J Neuroinflammation 15: 8, 2018.

23. Lässer C, Alikhani VS, Ekström K, Eldh M, Paredes PT, Bossios A, Sjöstrand M, Gabrielsson S, Lötvall J and Valadi H: Human saliva, plasma and breast milk exosomes contain RNA: Uptake by macrophages. J Transl Med 9: 9, 2011

24. Admyre C, Johansson SM, Qazi KR, Filén JJ, Lahesmaa R, Norman M, Neve EP, Scheynius A and Gabrielsson S: Exosomes with immune modulatory features are present in human breast milk. J Immunol 179: 1969-1978, 2007.

25. Street JM, Barran PE, Mackay CL, Weidt S, Balmforth C, Walsh TS, Chalmers RT, Webb DJ and Dear JW: Identification and proteomic profiling of exosomes in human cerebrospinal fluid. J Transl Med 10: 5, 2012.

26. Vojtech L, Woo S, Hughes S, Levy C, Ballweber L, Sauteraud RP, Strobl J, Westerberg K, Gottardo R, Tewari M and Hladik F: Exosomes in human semen carry a distinctive repertoire of small non-coding RNAs with potential regulatory functions. Nucleic Acids Res 42: 7290-7304, 2014.

27. Hong BS, Cho JH, Kim H, Choi EJ, Rho S, Kim J, Kim JH, Choi DS, Kim YK, Hwang D and Gho YS: Colorectal cancer cell-derived microvesicles are enriched in cell cycle-related mRNAs that promote proliferation of endothelial cells. BMC Genomics 10: 556, 2009.

28. Kumar B, Garcia M, Murakami JL and Chen CC: Exosome-mediated microenvironment dysregulation in leukemia. Biochim Biophys Acta 1863: 464-470, 2016.

29. Suetsugu A, Honma K, Saji S, Moriwaki H, Ochiya T and Hoffman RM: Imaging exosome transfer from breast cancer cells to stroma at metastatic sites in orthotopic nude-mouse models. Adv Drug Deliv Rev 65: 383-390, 2013.

30. Kogure T, Lin WL, Yan IK, Braconi C and Patel T: Intercellular nanovesicle-mediated microRNA transfer: A mechanism of environmental modulation of hepatocellular cancer cell growth. Hepatology 54: 1237-1248, 2011.

31. $\mathrm{Yu} \mathrm{S}, \mathrm{Cao} \mathrm{H}$, Shen B and Feng J: Tumor-derived exosomes in cancer progression and treatment failure. Oncotarget 6: 37151-37168, 2015.

32. Luga V and Wrana JL: Tumor-stroma interaction: Revealing fibroblast-secreted exosomes as potent regulators of Wnt-planar cell polarity signaling in cancer metastasis. Cancer Res 73: 6843-6847, 2013.

33. Shao Y, Shen Y, Chen T, Xu F, Chen X and Zheng S: The functions and clinical applications of tumor-derived exosomes. Oncotarget 7: 60736-60751,2016.

34. Corrado C, Raimondo S, Chiesi A, Ciccia F, De Leo G and Alessandro R: Exosomes as intercellular signaling organelles involved in health and disease: Basic science and clinical applications. Int J Mol Sci 14: 5338-5366, 2013.

35. Kowal J, Tkach M and Théry C: Biogenesis and secretion of exosomes. Curr Opin Cell Biol 29: 116-125, 2014.

36. Ekström EJ, Bergenfelz C, von Bülow V, Serifler F, Carlemalm E, Jönsson $\mathrm{G}$, Andersson T and Leandersson K: WNT5A induces release of exosomes containing pro-angiogenic and immunosuppressive factors from malignant melanoma cells. Mol Cancer 13: 88, 2014.

37. Man YG, Stojadinovic A, Mason J, Avital I, Bilchik A, Bruecher B, Protic $M$, Nissan A, Izadjoo $M$, Zhang $X$ and Jewett A: Tumor-infiltrating immune cells promoting tumor invasion and metastasis: Existing theories. J Cancer 4: 84-95, 2013.
38. Xue M, Zhuo Y and Shan B: MicroRNAs, long noncoding RNAs, and their functions in human disease. Methods Mol Biol 1617: $1-25,2017$.

39. Thomou T, Mori MA, Dreyfuss JM, Konishi M, Sakaguchi M, Wolfrum C, Rao TN, Winnay JN, Garcia-Martin R, Grinspoon SK, et al: Adipose-derived circulating miRNAs regulate gene expression in other tissues. Nature 542: 450-455, 2017.

40. Luga V, Zhang L, Viloria-Petit AM, Ogunjimi AA, Inanlou MR, Chiu E, Buchanan M, Hosein AN, Basik M and Wrana JL: Exosomes mediate stromal mobilization of autocrine Wnt-PCP signaling in breast cancer cell migration. Cell 151: 1542-1556, 2012.

41. van Balkom BW, de Jong OG, Smits M, Brummelman J, den Ouden K, de Bree PM, van Eijndhoven MA, Pegtel DM, Stoorvogel W, Würdinger T and Verhaar MC: Endothelial cellsrequire miR-214 to secrete exosomes that suppress senescence and induce angiogenesis in human and mouse endothelial cells. Blood 121: 3997-4006, 2013.

42. Cui Y, Luan J, Li H, Zhou X and Han J: Exosomes derived from mineralizing osteoblasts promote ST2 cell osteogenic differentiation by alteration of microRNA expression. FEBS Lett 590: 185-192, 2016.

43. Henderson MC and Azorsa DO: The genomic and proteomic content of cancer cellderived exosomes. Front Oncol 2: 38, 2012.

44. Nolte-'t Hoen EN, Buermans HP, Waasdorp M, Stoorvogel W, Wauben MH and 't Hoen PA: Deep sequencing of RNA from immune cell-derived vesicles uncovers the selective incorporation of small non-coding RNA biotypes with potential regulatory functions. Nucleic Acids Res 40: 9272-9285, 2012.

45. Cazzoli R, Buttitta F, Di Nicola M, Malatesta S, Marchetti A, Rom WN and Pass HI: microRNAs derived from circulating exosomes as noninvasive biomarkers for screening and diagnosing lung cancer. J Thorac Oncol 8: 1156-1162, 2013.

46. Corcoran C, Friel AM, Duffy MJ, Crown J and O'Driscoll L: Intracellular and extracellular microRNAs in breast cancer. Clin Chem 57: 18-32, 2011

47. Paget S: The distribution of secondary growths in cancer of the breast. 1889 Cancer Metastasis Rev 8: 98-101, 1889.

48. Brennan MF and Ekman L: Metabolic consequences of nutritional support of the cancer patient. Cancer 54 (11 Suppl): S2627-S2634, 1984.

49. Guise TA, Mohammad KS, Clines G, Stebbins EG, Wong DH, Higgins LS, Vessella R, Corey E, Padalecki S, Suva L and Chirgwin JM: Basic mechanisms responsible for osteolytic and osteoblastic bone metastases. Clin Cancer Res 12: 6213s-6216s, 2006.

50. Kingsley LA, Fournier PG, Chirgwin JM and Guise TA: Molecular biology of bone metastasis. Mol Cancer Ther 6: 2609-2617, 2007

51. Pantel K, Müller V, Auer M, Nusser N, Harbeck N and Braun S Detection and clinical implications of early systemic tumor cell dissemination in breast cancer. Clin Cancer Res 9: 6326-6334, 2003.

52. Aft R, Naughton M, Trinkaus K, Watson M, Ylagan L, Chavez-Macgregor M, Zhai J, Kuo S, Shannon W, Diemer K, et al: Effect of zoledronic acid on disseminated tumour cells in women with locally advanced breast cancer: An open label, randomised, phase 2 trial. Lancet Oncol 11: 421-428, 2010.

53. Chen H, Senda T and Kubo KY: The osteocyte plays multiple roles in bone remodeling and mineral homeostasis. Med Mo Morphol 48: 61-68, 2015.

54. Nguyen DX, Bos PD and Massagué J: Metastasis: From dissemination to organ-specific colonization. Nat Rev Cancer 9: 274-284, 2009.

55. Saleem SN and Abdel-Mageed AB: Tumor-derived exosomes in oncogenic reprogramming and cancer progression. Cell Mol Life Sci 72: 1-10, 2015.

56. Clézardin P: The role of RANK/RANKL/osteoprotegerin (OPG) triad in cancer-induced bone diseases: Physiopathology and clinical implications. Bull Cancer 98: 837-846, 2011 (In French).

57. Sanders JL, Chattopadhyay N, Kifor O, Yamaguchi T, Butters RR and Brown EM: Extracellular calcium-sensing receptor expression and its potential role in regulating parathyroid hormone-related peptide secretion in human breast cancer cell lines. Endocrinology 141: 4357-4364, 2000.

58. Siveen KS, Prabhu K, Krishnankutty R, Kuttikrishnan S, Tsakou M, Alali FQ, Dermime S, Mohammad RM and Uddin S: Vascular endothelial growth factor (VEGF) signaling in tumour vascularization: Potential and challenges. Curr Vasc Pharmacol 15: 339-351, 2017. 
59. Heldin $\mathrm{CH}$, Lennartsson $\mathrm{J}$ and Westermark B: Involvement of platelet-derived growth factor ligands and receptors in tumorigenesis. J Intern Med 283: 16-44, 2018.

60. Chirgwin JM, Mohammad KS and Guise TA: Tumor-bone cellular interactions in skeletal metastases. J Musculoskeletal Neuronal Interact 4: 308-318, 2004.

61. Chang AC, Chen PC, Lin YF, Su CM, Liu JF, Lin TH, Chuang SM and Tang CH: Osteoblast-secreted WISP-1 promotes adherence of prostate cancer cells to bone via the VCAM-1/integrin $\alpha 4 \beta 1$ system. Cancer Lett 426: 47-56, 2018.

62. D'Oronzo S, Brown J and Coleman R: The role of biomarkers in the management of bone-homing malignancies. J Bone Oncol 9: $1-9,2017$.

63. Berruti A, Libè R, Laganà M, Ettaieb H, Sukkari MA Bertherat J, Feelders RA, Grisanti S, Cartry J, Mazziotti G, et al: Morbidity and mortality of bone metastases in advanced adrenocortical carcinoma: A multicenter retrospective study. Eur J Endocrinol 180: 311-320, 2019.

64. Liao EY, Zhang ZL, Xia WB, Lin H, Cheng Q, Wang L, Hao YQ, Chen DC, Tang H, Peng YD, et al: Clinical characteristics associated with bone mineral density improvement after 1-year alendronate/vitamin $\mathrm{d} 3$ or calcitriol treatment: Exploratory results from a phase 3 , randomized, controlled trial on postmenopausal osteoporotic women in China. Medicine (Baltimore) 97: e11694, 2018

65. Stopeck AT, Lipton A, Body JJ, Steger GG, Tonkin K, de Boer RH, Lichinitser M, Fujiwara Y, Yardley DA, Viniegra M, et al: Denosumab compared with zoledronic acid for the treatment of bone metastases in patients with advanced breast cancer: A randomized, double-blind study. J Clin Oncol 28: 5132-5139, 2010

66. Body JJ, Lipton A, Gralow J, Steger GG, Gao G, Yeh H and Fizazi K: Effects of denosumab in patients with bone metastases with and without previous bisphosphonate exposure. J Bone Miner Res 25: 440-446, 2010.

67. Fizazi K, Lipton A, Mariette X, Body JJ, Rahim Y, Gralow JR Gao G, Wu L, Sohn W and Jun S: Randomized phase II trial of denosumab in patients with bone metastases from prostate cancer, breast cancer, or other neoplasms after intravenous bisphosphonates. J Clin Oncol 27: 1564-1571, 2009.

68. Hirbe A, Morgan EA, Uluçkan O and Weilbaecher K: Skeletal complications of breast cancer therapies. Clin Cancer Res 12: 6309s-6314s, 2006.

69. Fizazi K, Carducci M, Smith M, Damião R, Brown J, Karsh L, Milecki P, Shore N, Rader M, Wang H, et al: Denosumab versus zoledronic acid for treatment of bone metastases in men with castration-resistant prostate cancer: A randomised, double-blind study. Lancet 377: 813-822, 2011.

70. Zeng Z, Li Y, Pan Y, Lan X, Song F, Sun J, Zhou K, Liu X, Ren X, Wang F, et al: Cancer-derived exosomal miR-25-3p promotes pre-metastatic niche formation by inducing vascular permeability and angiogenesis. Nat Commun 9: 5395, 2018.

71. Sethi N and Kang Y: Unravelling the complexity of metastasis-molecular understanding and targeted therapies. Nat Rev Cancer 11: 735-748, 2011.

72. Kaplan RN, Riba RD, Zacharoulis S, Bramley AH, Vincent L, Costa C, MacDonald DD, Jin DK, Shido K, Kerns SA, et al: VEGFR1-positive haematopoietic bone marrow progenitors initiate the pre-metastatic niche. Nature 438 : 820-827, 2005

73. Psaila B and Lyden D: The metastatic niche: Adapting the foreign soil. Nat Rev Cancer 9: 285-293, 2009.

74. O'Brien K, Rani S, Corcoran C, Wallace R, Hughes L, Friel AM, McDonnell S, Crown J, Radomski MW and O'Driscoll L: Exosomes from triple-negative breast cancer cells can transfer phenotypic traits representing their cells of origin to secondary cells. Eur J Cancer 49: 1845-1859, 2013.

75. Kahlert $\mathrm{C}$ and Kalluri R: Exosomes in tumor microenvironment influence cancer progression and metastasis. J Mol Med (Berl) 91: 431-437, 2013.

76. Logozzi M, De Milito A, Lugini L, Borghi M, Calabrò L, Spada M, Perdicchio M, Marino ML, Federici C, Iessi E, et al: High levels of exosomes expressing CD63 and caveolin-1 in plasma of melanoma patients. PLoS One 4: e5219, 2009.

77. Tavoosidana G, Ronquist G, Darmanis S, Yan J, Carlsson L, Wu D, Conze T, Ek P, Semjonow A, Eltze E, et al: Multiple recognition assay reveals prostasomes as promising plasma biomarkers for prostate cancer. Proc Natl Acad Sci USA 108: 8809-8814, 2011.

78. Steinbichler TB, Dudás J, Riechelmann H and Skvortsovab II: The role of exosomes in cancer metastasis. Semin Cancer Biol 44: 170-181, 2017.
79. Gao D, Nolan D, McDonnell K, Vahdat L, Benezra R, Altorki N and Mittal V: Bone marrow-derived endothelial progenitor cells contribute to the angiogenic switch in tumor growth and metastatic progression. Biochim Biophys Acta 1796: 33-40, 2009.

80. Erler JT, Bennewith KL, Cox TR, Lang G, Bird D, Koong A, Le QT and Giaccia AJ: Hypoxia-induced lysyl oxidase is a critical mediator of bone marrow cell recruitment to form the premetastatic niche. Cancer Cell 15: 35-44, 2009.

81. Hiratsuka S, Watanabe A, Aburatani $H$ and Maru Y: Tumour-mediated upregulation of chemoattractants and recruitment of myeloid cells predetermines lung metastasis. Nat Cell Biol 8: 1369-1375, 2006 .

82. Han C, Zhou J, Liu B, Liang C, Pan X, Zhang Y, Zhang Y, Wang Y, Shao L, Zhu B, et al: Delivery of miR-675 by stem cell-derived exosomes encapsulated in silk fibroin hydrogel prevents aging-induced vascular dysfunction in mouse hindlimb. Mater Sci Eng C Mater Biol Appl 99: 322-332, 2019.

83. Wiklander OP, Nordin JZ, O'Loughlin A, Gustafsson Y, Corso G, Mäger I, Vader P, Lee Y, Sork H, Seow Y, et al: Extracellular vesicle in vivo biodistribution is determined by cell source, route of administration and targeting. J Extracell Vesicles 4: 26316, 2015.

84. Peinado H, Alečković M, Lavotshkin S, Matei I, Costa-Silva B, Moreno-Bueno G, Hergueta-Redondo M, Williams C, García-Santos G, Ghajar C, et al: Melanoma exosomes educate bone marrow progenitor cells toward a pro-metastatic phenotype through MET. Nat Med 18: 883-891, 2012.

85. Hoshino A, Costa-Silva B, Shen TL, Rodrigues G, Hashimoto A, Tesic Mark M, Molina H, Kohsaka S, Di Giannatale A, Ceder S, et al: Tumour exosome integrins determine organotropic metastasis. Nature 527: 329-335, 2015.

86. Peinado H, Lavotshkin S and Lyden D: The secreted factors responsible for pre-metastatic niche formation: Old sayings and new thoughts. Semin Cancer Biol 21: 139-146, 2011.

87. Hameed A, Brady JJ, Dowling P, Clynes M and O'Gorman P: Bone disease in multiple myeloma: Pathophysiology and management. Cancer Growth Metastasis 7: 33-42, 2014.

88. Abdi J, Chen $G$ and Chang H: Drug resistance in multiple myeloma: Latest findings and new concepts on molecular mechanisms. Oncotarget 4: 2186-2207, 2013.

89. Rossi M, Pitari MR, Amodio N, Di Martino MT, Conforti F, Leone E, Botta C, Paolino FM, Del Giudice T, Iuliano E, et al: miR-29b negatively regulates human osteoclastic cell differentiation and function: Implications for the treatment of multiple myeloma-related bone disease. J Cell Physiol 228: 1506-1515, 2013.

90. Heider U, Fleissner C, Zavrski I, Kaiser M, Hecht M, Jakob C and Sezer O: Bone markers in multiple myeloma. Eur J Cancer 42: 1544-1553, 2006

91. Raimondi L, De Luca A, Amodio N, Manno M, Raccosta S, Taverna S, Bellavia D, Naselli F, Fontana S, Schillaci O, et al: Involvement of multiple myeloma cell-derived exosomes in osteoclast differentiation. Oncotarget 6: 13772-13789, 2015.

92. Garimella R, Washington L, Isaacson J, Vallejo J, Spence M, Tawfik O, Rowe P, Brotto M and Perez R: Extracellular membrane vesicles derived from 143B osteosarcoma cells contain pro-osteoclastogenic cargo: A novel communication mechanism in osteosarcoma bone microenvironment. Transl Oncol 7: 331-340, 2014.

93. Raimondo S, Saieva L, Vicario E, Pucci M, Toscani D, Manno M, Raccosta S, Giuliani N and Alessandro R: Multiple myeloma-derived exosomes are enriched of amphiregulin (AREG) and activate the epidermal growth factor pathway in the bone microenvironment leading to osteoclastogenesis. J Hematol Oncol 12: 2, 2019.

94. Deng X, He G, Liu J, Luo F, Peng X, Tang S, Gao Z, Lin Q, Keller JM, Yang T and Keller ET: Recent advances in bone-targeted therapies of metastatic prostate cancer. Cancer Treat Rev 40: 730-738, 2014.

95. Karlsson T, Lundholm M, Widmark A and Persson E: Tumor cell-derived exosomes from the prostate cancer cell line TR AMP-C1 impair osteoclast formation and differentiation. PLoS One 11: e0166284, 2016.

96. Inder KL, Ruelcke JE, Petelin L, Moon H, Choi E, Rae J, Blumenthal A, Hutmacher D, Saunders NA, Stow JL, et al: Cavin-1/PTRF alters prostate cancer cell-derived extracellular vesicle content and internalization to attenuate extracellular vesicle-mediated osteoclastogenesis and osteoblast proliferation. J Extracell Vesicles 3, 2014 
97. Shiozawa Y, Pedersen EA, Havens AM, Jung Y, Mishra A, Joseph J, Kim JK, Patel LR, Ying C, Ziegler AM, et al: Human prostate cancer metastases target the hematopoietic stem cell niche to establish footholds in mouse bone marrow. J Clin Invest 121: 1298-1312, 2011.

98. Morrissey C, Lai JS, Brown LG, Wang YC, Roudier MP, Coleman IM, Gulati R, Vakar-Lopez F, True LD, Corey E, et al: The expression of osteoclastogenesis-associated factors and osteoblast response to osteolytic prostate cancer cells. Prostate 70: 412-424, 2010.

99. Itoh T,Ito Y, Ohtsuki Y, Ando M,Tsukamasa Y, Yamada N,Naoe T and Akao Y: Microvesicles released from hormone-refractory prostate cancer cells facilitate mouse pre-osteoblast differentiation. J Mol Histol 43: 509-515, 2012.

100. Ye Y, Li SL, Ma YY, Diao YJ, Yang L, Su MQ, Li Z, Ji Y, Wang J, Lei L, et al: Exosomal miR-141-3p regulates osteoblast activity to promote the osteoblastic metastasis of prostate cancer. Oncotarget 8: 94834-94849, 2017.

101. Hashimoto K, Ochi H, Sunamura S, Kosaka N, Mabuchi Y, Fukuda $\mathrm{T}$, Yao $\mathrm{K}$, Kanda $\mathrm{H}$, Ae K, Okawa A, et al: Cancer-secreted hsa-miR-940 induces an osteoblastic phenotype in the bone metastatic microenvironment via targeting ARHGAP1 and FAM134A. Proc Natl Acad Sci USA 115: 2204-2209, 2018.

102. Morhayim J, van de Peppel J, Demmers JA, Kocer G, Nigg AL, van Driel $\mathrm{M}$, Chiba $\mathrm{H}$ and van Leeuwen JP: Proteomic signatures of extracellular vesicles secreted by nonmineralizing and mineralizing human osteoblasts and stimulation of tumor cell growth. FASEB J 29: 274-285, 2015.
103. Peng X, Guo W, Ren T, Lou Z, Lu X, Zhang S, Lu Q and Sun Y: Differential expression of the RANKL/RANK/OPG system is associated with bone metastasis in human non-small cell lung cancer. PLoS One 8: e58361, 2013.

104. Taverna S, Pucci M, Giallombardo M, Di Bella MA, Santarpia M, Reclusa P, Gil-Bazo I, Rolfo C and Alessandro R: Amphiregulin contained in NSCLC-exosomes induces osteoclast differentiation through the activation of EGFR pathway. Sci Rep 7: 3170, 2017.

105. Valencia K, Luis-Ravelo D, Bovy N, Antón I, Martínez-Canarias S, Zandueta C, Ormazábal C, Struman I, Tabruyn S, Rebmann V, et al: miRNA cargo within exosome like vesicle transfer influences metastatic bone colonization. Mol Oncol 8: 689-703, 2014.

106. Kumar B, Garcia M, Weng L, Jung X, Murakami JL, Hu X, McDonald T, Lin A, Kumar AR, DiGiusto DL, et al: Acute myeloid leukemia transforms the bone marrow niche into a leukemia-permissive microenvironment through exosome secretion. Leukemia 32: 575-587, 2018.

107. Guise TA: Breast cancer bone metastases: It's all about the neighborhood. Cell 154: 957-959, 2013.

(i) $($ ) This work is licensed under a Creative Commons Attribution-NonCommercial-NoDerivatives 4.0 International (CC BY-NC-ND 4.0) License. 